\title{
GABA and glycine immunoreactivity in the guinea pig superior olivary complex
}

\author{
Robert H. Helfert ${ }^{1}$, Joann M. Bonneau ${ }^{1}$, Robert J. Wenthold ${ }^{2}$ and \\ Richard A. Altschuler ${ }^{1}$ \\ ${ }^{1}$ Kresge Hearing Research Institute, The University of Michigan, Ann Arbor, MI 48109 (U.S.A.) and ${ }^{2}$ Laboratory of \\ Neuro-otolaryngology, NINCDS, NIH, Bethesda, MD 20205 (U.S.A.)
}

(Accepted 4 April 1989)

Key words: Lateral superior olive; Medial superior olive; Medial nucleus of the trapezoid body; Periolivary nuclei; Immunocytochemistry

\begin{abstract}
Immunoperoxidase immunocytochemistry was employed to examine the distribution of $\gamma$-aminobutyric acid (GABA)- and glycine (GLY)-immunoreactive cells, fibers, and terminals in the guinea pig superior olivary complex. The nuclei studied were the lateral superior olive (LSO), medial superior olive (MSO), superior paraolivary nucleus (SPN), and the medial, ventral, and lateral nuclei of the trapezoid body (MNTB, VNTB, and LNTB, respectively). The majority of LSO neurons exhibited GABA-immunoreactive (+) labeling. These same neurons were also lightly GLY+. Extensive perisomatic punctate GLY+ labeling was observed on most LSO neurons; these puncta most likely correspond to synaptic terminals. A very small number of MSO fusiform neurons were GABA+, and none were GLY+. The GLY positive perisomatic punctate labeling around most MSO neurons, although abundant, was not as profuse as that observed in the LSO. The MNTB neurons corresponding to the principal and elongate types were intensely GLY+ and were contacted by small numbers of GLY+ puncta. There was extensive GLY+ punctate labeling in the SPN that surrounded the cell bodies of most of its large, radiate neurons and many of the smaller, fusiform neurons. The few large, radiate neurons that were lightly GLY+ possessed far fewer GLY + puncta on their perikarya. The distribution of GABA+ puncta was generally diffuse and scattered throughout the nuclei described above. In the VNTB and LNTB, several large neurons of various shapes were GLY+ as were the small, oval neurons. The extent of GLY+ punctate labeling was quite variable in both nuclei. The majority of perikarya in the VNTB and LNTB were GABA+. A light distribution of GABA+ puncta was observed on most cell bodies in both nuclei. Peridendritic GABA + punctate labeling was dense in the VNTB neuropil. Two small populations of GLY+ neurons were observed outside of the named nuclei of the SOC; one was located dorsal to the LSO, near its dorsal hilus, and the other was identified near the medial pole of the LSO. The somata of both populations possessed extremely sparse GLY+ punctate labeling. In general, these results agree with and expand on findings in rodents from previous studies. There appears, however, to be differences between the guinea pig and cat with regard to the proportions of GABA+ neurons in the LSO and GLY+ punctate labeling in the MSO.
\end{abstract}

\section{INTRODUCTION}

In recent years, substantial effort has been devoted to localizing and determining the functional roles of $\gamma$-aminobutyric acid (GABA) and glycine (GLY) in nuclear complexes of the auditory brainstem $^{3,9,10,13,25,26,30,43,44,65,71,72,76,81,89,91,101,102}$.

This effort evolved from physiological studies describing the extensive involvement of inhibition in the processing of acoustic information $7,27,28,37,45$,
46,79,93, and the evidence supporting GABA and GLY as major transmitters mediating inhibition in the central nervous system ${ }^{14,29,75,90,103}$.

The goal of this study is to describe, in detail, the patterns of GABA- and GLY-immunoreactive cells, fibers, and puncta in the nuclei composing the superior olivary complex (SOC). The mammalian SOC, which contains major relay nuclei of the auditory system ${ }^{48,59,73,95}$, is composed of 3 principal nuclei: the lateral superior olive (LSO), medial 
superior olive (MSO), and medial nucleus of the trapezoid body (MNTB). They are surrounded by smaller aggregates of neurons known, collectively, as the periolivary nuclei (PON). In rodents, the PON have been named according to their location in the superior olivary complex as follows: the ventral nucleus of the trapezoid body (VNTB) is ventral to the MNTB, the lateral nucleus of the trapezoid body (LNTB) is lateral to the VNTB and ventrolateral to the LSO, and the superior paraolivary nucleus (SPN) is dorsal to MNTB. The LSO and MSO are initial sites for the convergence of binaural input ${ }^{78,88}$ and are probably involved in localizing sounds ${ }^{37}$. 62,63,93,107; the MNTB assists in this function by providing the LSO with input from the contralateral ear $^{32,86,88,98}$. The projection from the MNTB to the LSO is considered to be inhibitory ${ }^{65}$. The PON possess complex patterns of neural connectivity, and contain elements of both the ascending and descending auditory pathways to be discussed below.

Several immunocytochemical studies have described the distribution of GABA- or GLY-immunoreactivity in selected nuclei in the SOC as a part of a broader examination of the auditory brainstem of rats ${ }^{13}$ and guinea pigs ${ }^{71,72,91,102}$. In doing so, the evaluation of several SOC nuclei, in particular the PON, was either not attempted or only briefly described. The present study examined the immunoreactive labeling of GABA and GLY in all of the nuclei composing the guinea pig superior olivary complex.

\section{MATERIALS AND METHODS}

Thirty NIH-strain guinea pigs, $200-300 \mathrm{~g}$, were used in this study. All of the animals were deeply anesthetized with pentobarbital, exsanguinated transcardially with $50 \mathrm{ml}$ of physiological saline containing $0.01 \%$ sodium nitrite, and perfused with $750 \mathrm{ml}$ of mixed-aldehyde fixative in $0.1 \mathrm{M}$ sodium cacodylate buffer $(\mathrm{pH} 7.3-7.4)$ delivered over a 15-min time period. The fixative contained $1.25 \%$ glutaraldehyde and $1.0 \%$ paraformaldehyde for the 14 animals used in GLY immunocytochemical studies; $4.0 \%$ paraformaldehyde and $0.2 \%$ glutaraldehyde for those 14 evaluated for GABA immunoreactivity; and $3 \%$ paraformaldehyde and $0.5 \%$ glutaraldehyde for 2 animals whose tissues were examined for possible co-localization of GABA and GLY antibodies. The brainstems were then removed, immersed in their respective fixatives for an additional $2-4 \mathrm{~h}$, and stored overnight at $4{ }^{\circ} \mathrm{C}$ in $300-310$ mOsm phosphate-buffered saline (PBS), $\mathrm{pH} 7.4$.

Serial transverse sections of the brainstems from 9 animals of each of the GABA and GLY groups were cut at $60 \mu \mathrm{m}$ on a Vibratome. After washing in PBS, the free-floating sections were pre-incubated 1 $\mathrm{h}$ in a blocking solution of $5 \%$ normal goat serum (NGS) in PBS. They were then transferred to affinity-purified polyclonal rabbit antisera to either GLY ${ }^{102}$, diluted 1:1000-1:1250, or $\mathrm{GABA}^{101}$, diluted 1:1500-1:1750, and incubated overnight at $4{ }^{\circ} \mathrm{C}$ with gentle agitation. The antisera dilutions were made using blocking solution as the diluent. Control sections were incubated in blocking solution that did not contain the primary antisera. Subsequent steps followed the avidin-biotin method of Hsu et al. ${ }^{56}$ using the Vectastain ABC kit (Vector Labs), and the peroxidase activity was visualized by exposing the sections to $0.05 \%$ diaminobenzidine and $0.0075-0.001 \%$ hydrogen peroxide in PBS for 5-15 min. The sections were finally washed in distilled water $\left(\mathrm{dH}_{2} \mathrm{O}\right)$, mounted onto gelatinsubbed slides, and coverslipped with Krystalon mounting medium (Harleco).

For post-embedding immunocytochemistry the brainstems from the remaining animals were Vibratome-sectioned at $100-150 \mu \mathrm{m}$ in the transverse plane. The tissue was post-fixed in $0.2 \%$ osmium tetroxide in $0.15 \mathrm{M}$ sodium cacodylate buffer $(\mathrm{pH}$ 7.4) for $2 \mathrm{~h}$, dehydrated through a graded ethanol series and propylene oxide, and embedded in EMbed 812-based resin (Electron Microscopy Supplies). The embedded tissue was sectioned at $1 \mu \mathrm{m}$ with an ultramicrotome (Reichert Ultracut or LKB Ultratome) and mounted onto glass microscope slides. To allow the antiserum better penetration into the tissue, the plastic was removed from the sections by an $11 \mathrm{~min}$ immersion into saturated sodium ethoxide (absolute ethanol saturated with sodium hydroxide) diluted $1: 1$ with absolute ethanol, followed by $\mathrm{dH}_{2} \mathrm{O}$ washes. To restore antigenicity, osmium was bleached from the tissue by two 5-min submersions into $1 \%$ aqueous sodium $m$-periodate, separated by a brief $\mathrm{dH}_{2} \mathrm{O}$ wash. Following additional $\mathrm{dH}_{2} \mathrm{O}$ rinses, the tissue was pretreated in blocking solution 
(5\% NGS in PBS) and incubated in a humidified chamber, at $4{ }^{\circ} \mathrm{C}$, for $48 \mathrm{~h}$ in the appropriate primary antibody dilution. GLY and GABA antisera were diluted in blocking solution 1:400 and 1:500, respectively. Control sections were incubated in blocking solution only. The procedures for visualizing the immunoreactivity were the same as in the preceding paragraphs, except that the Vectastain reagents were prepared at twice the recommended concentration.

For the tissue prepared for evaluation of GABA and GLY co-localization, adjacent $1 \mu \mathrm{m}$ plastic sections through the SOC were processed immunocytochemically as described in the preceding paragraph. One section was incubated in GABA antisera, the other in GLY antisera. The coincidence of GABA and GLY immunoreactivity within a given SOC profile was assessed by comparing photomontages of the adjacent sections. From this method, evidence for GABA and GLY co-localization could be obtained.

The material for this study was examined with a
Leitz Dialux equipped with both bright-field and differential interference contrast optics.

\section{RESULTS}

Each of the two immunolabeling methods employed offered distinct advantages in terms of interpreting immunoreactive labeling. Processing the tissue as free-floating sections produced better immunolabeling of perikarya and dendrites (e.g. Fig. 1) than that observed in the $1 \mu \mathrm{m}$ plastic-embedded sections due, perhaps, to the greater tissue depth available in the thicker free-floating sections. However, the labeling of small perisomatic and peridendritic puncta was more intense in the plasticembedded tissue (e.g. Figs. 2, 3).

No immunoprecipitate was observed in the control tissues regardless of whether they were processed as free-floating sections or by the postembedding technique. In sections exposed to the primary antisera, a range of labeling intensities
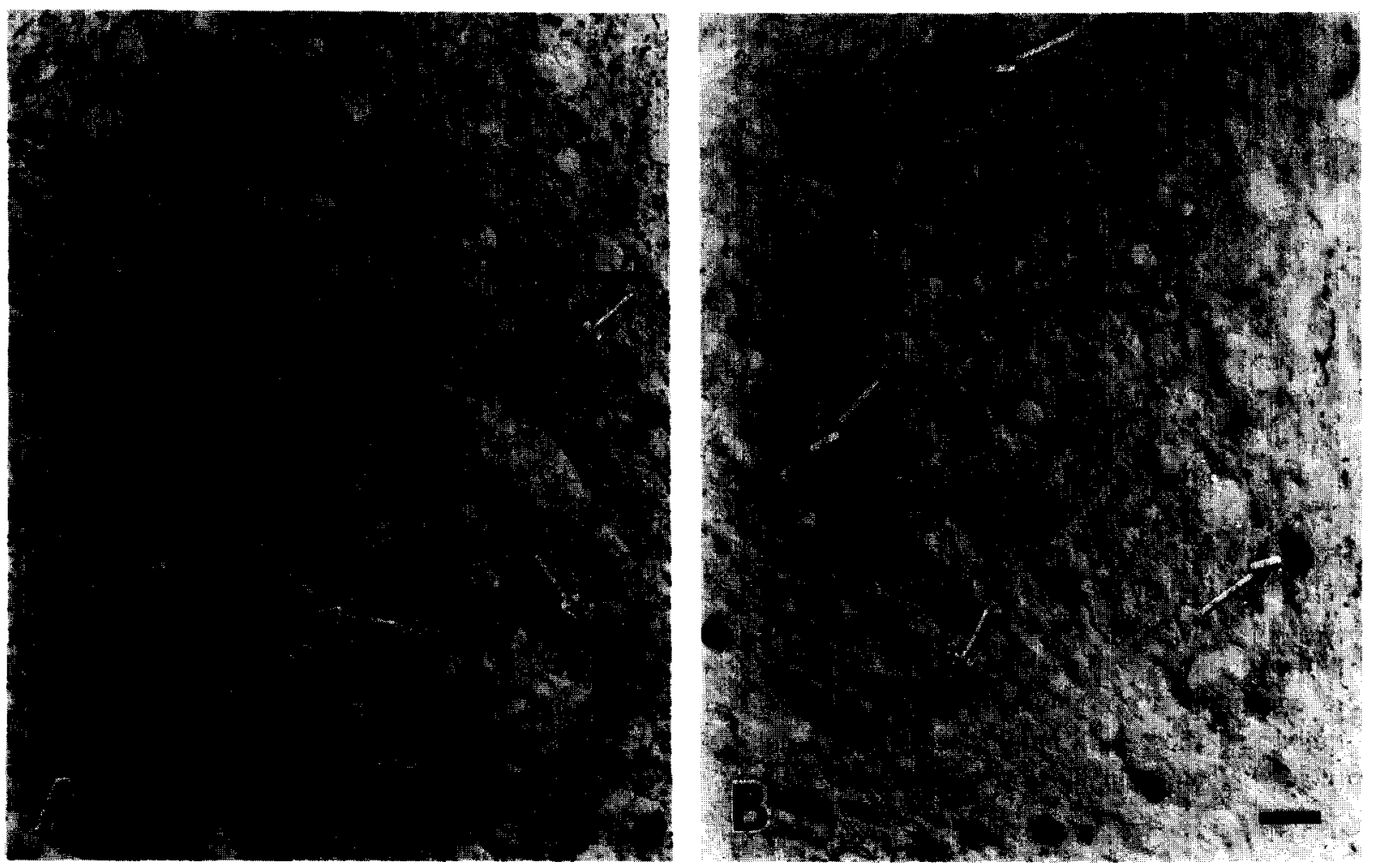

Fig. 1. GLY and GABA immunolabeling in the LSO. A: the neuropil of the LSO is rich with GLY immunolabeled fibers and puncta. Arrows indicate examples of LSO neurons that are lightly GLY immunopositive. B: arrows indicate LSO neurons that are intensely GABA immunoreactive. Bar $=20 \mu \mathrm{m}$. 

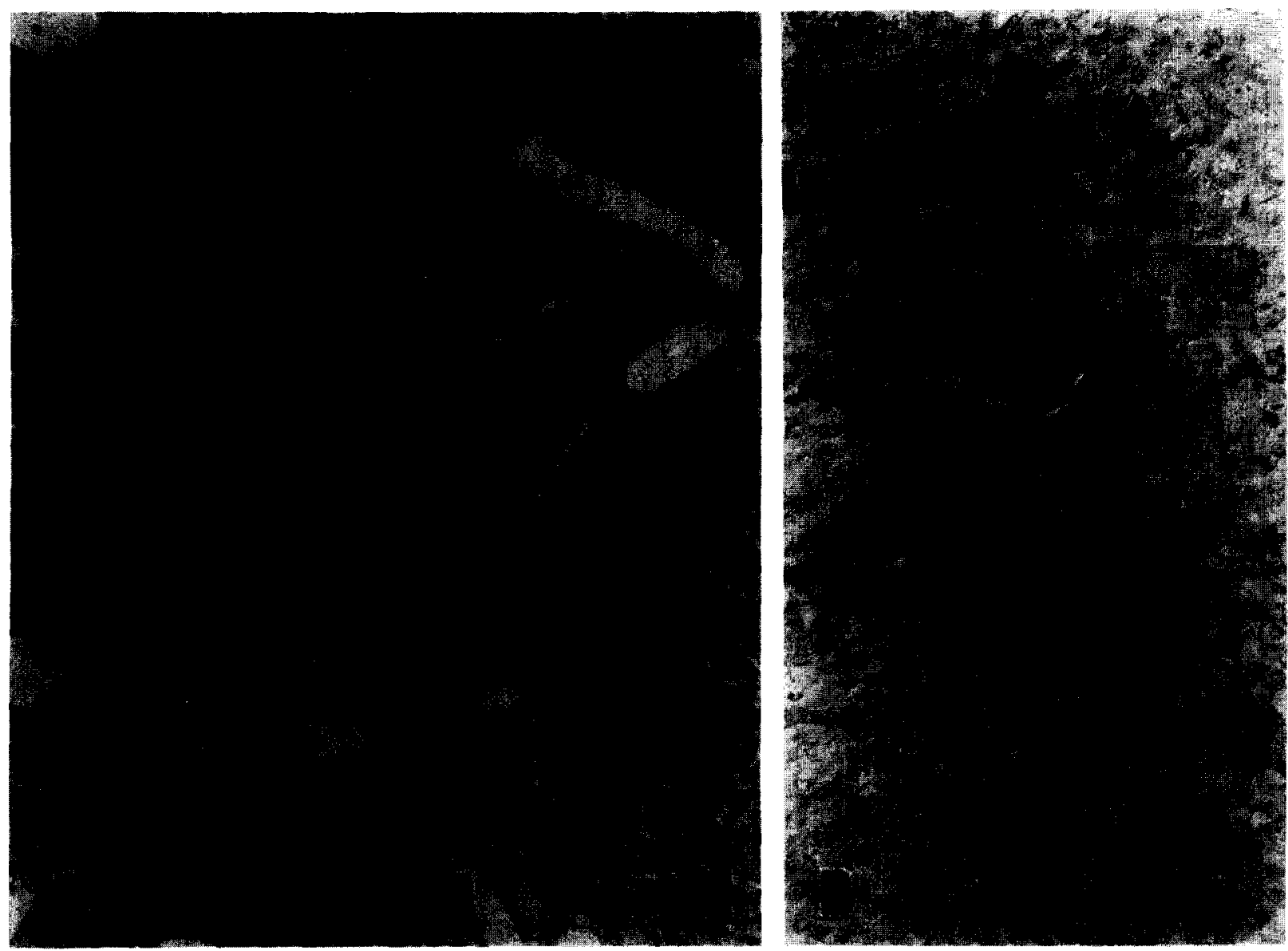

Fig. 2. Co-localization of GLY and GABA immunoreactivity in LSO neurons. The same field is seen in adjacent $1 \mu \mathrm{m}$ section immunolabeled with GLY (A) or GABA (B); arrows indicate immunopositive somata, arrowheads indicate unlabeled ones. Note that the GLY immunopositive neurons in A are the same as those that are GABA immunoreactive in $\mathrm{B}$. In $\mathrm{A}$ note also that both labeled and unlabeled perikarya are encrusted with GLY immunopositive puncta. Bar $=20 \mu \mathrm{m}$.

occurred that presumably corresponded, qualitatively, to the amount of antigen (GLY or GABA) contained within the labeled profiles. Axons were typically the most intensely immunostained structures. While synaptic terminals cannot be unambiguously identified at the light microscopic level, the labeled puncta observed most likely corresponded to terminals or axons en passant. When labeled puncta were encountered on somata or dendrites, it was assumed that those with immunoreactivities similar to nearby longitudinally-sectioned labeled axons were indeed axonal, while puncta more lightly stained were presynaptic terminals (Fig. 4A).

For the sake of convenience, GABA- and GLYlike immunoreactivity will be referred to as GABA+ and GLY+, respectively.

\section{Lateral superior olivary nucleus}

Two-thirds to three-quarters of the LSO neuronal population exhibited light GLY immunoreactivity; the rest were unlabeled (Fig. 1A). Similar proportions of LSO neurons were distinctly GABA+ while

Fig. 3. Punctate GLY and GABA labeling in the LSO. A: the profiles of most LSO neuronal somata are surrounded by GLY immunoreactive puncta (examples of which are indicated by the arrows), most of which are probably presynaptic terminals. B: punctate GABA immunoreactive labeling in the LSO is sparse. Arrows indicate examples of GABA immunopositive puncta on the cell bodies of GABA immunostained LSO neurons. Bar $=20 \mu \mathrm{m}$. 


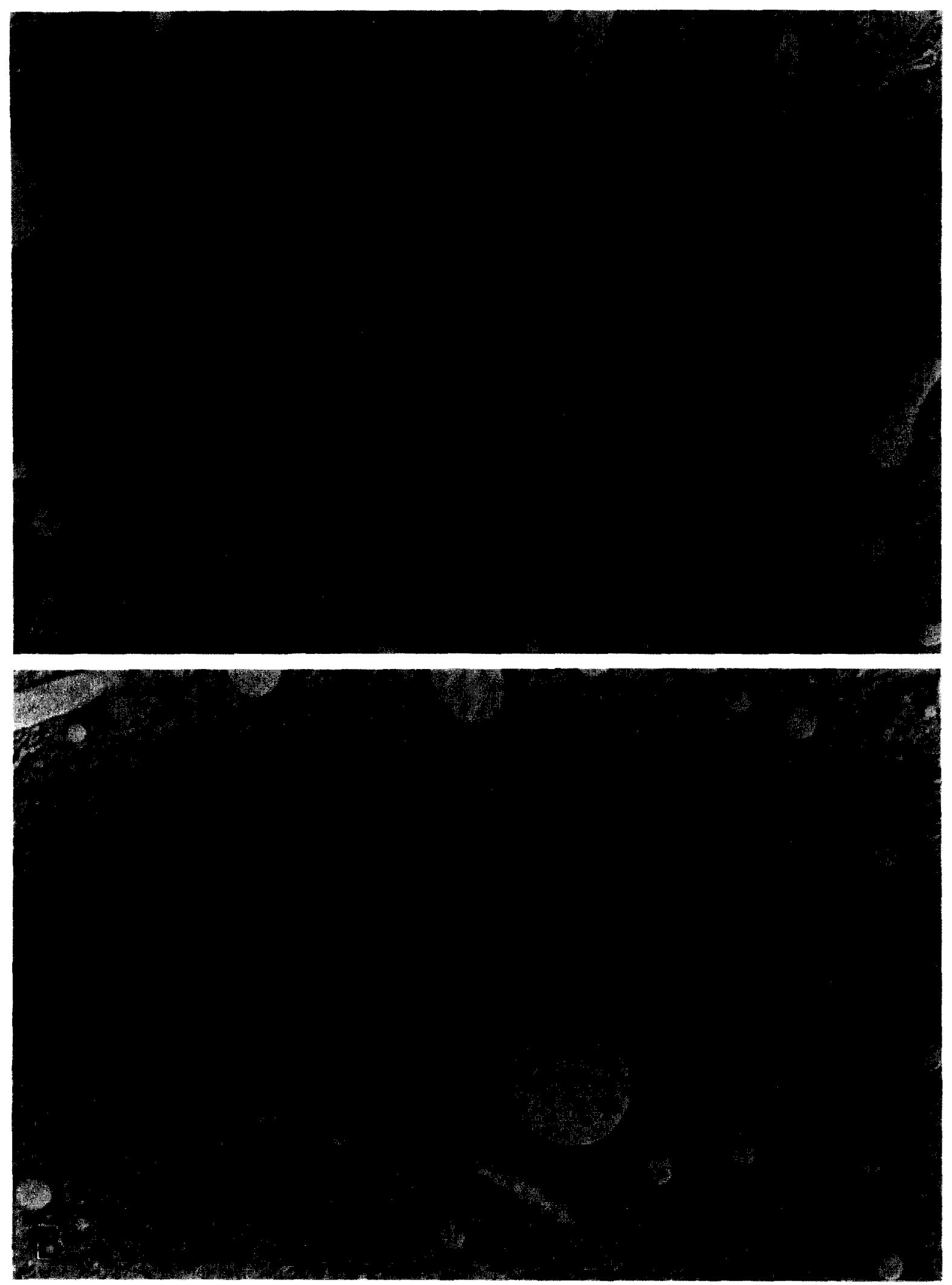



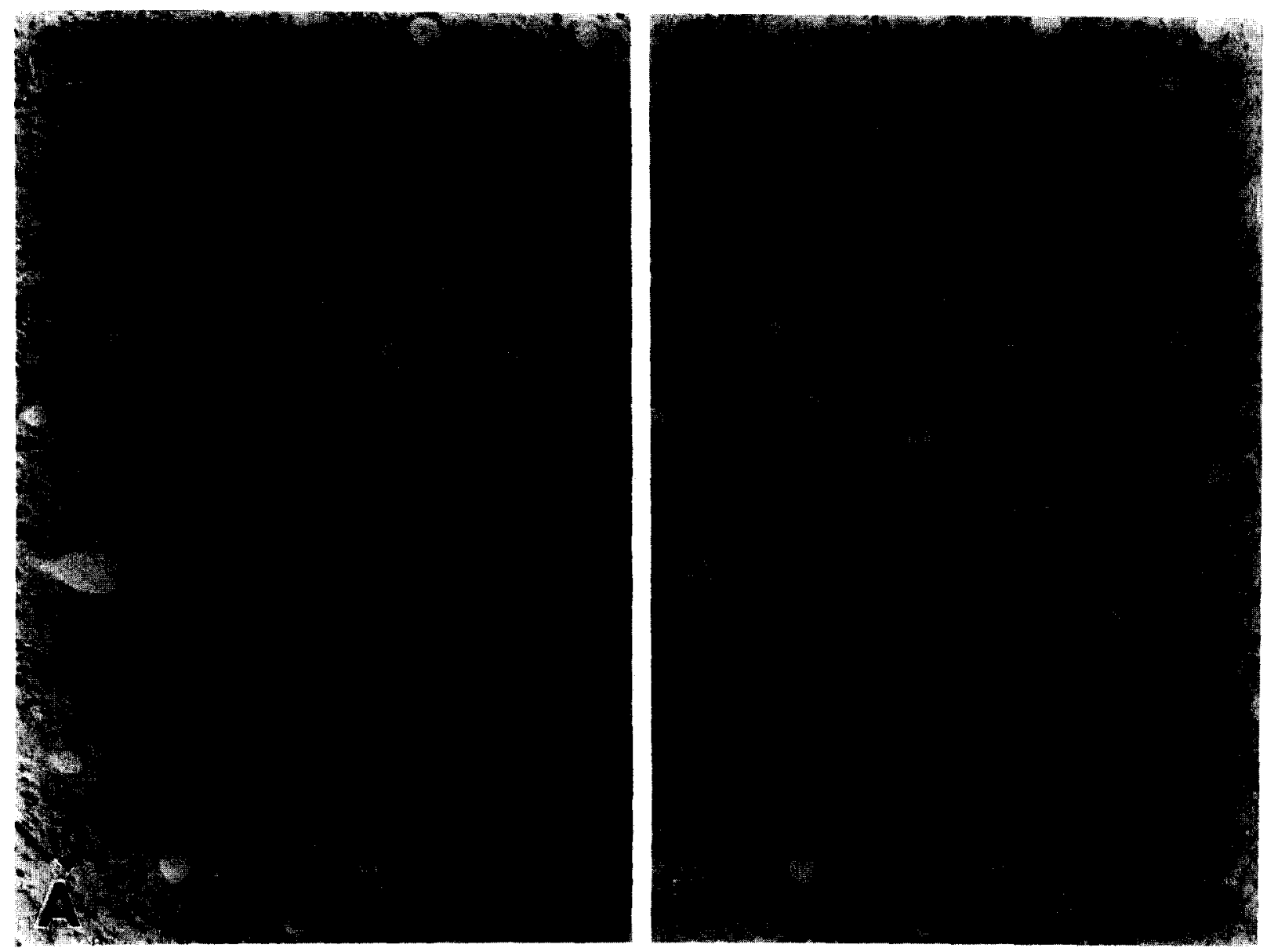

Fig. 4. GLY and GABA immunolabeling in the MSO. A: the MSO contains abundant GLY immunoreactive fibers and puncta. The large arrowheads indicate examples of punctate labeling, the intensity of which is typical for axons; the arrows indicate examples of punctate labeling that are presumed to correspond to presynaptic terminals. B: arrow indicates an intensely GABA immunopositive MSO neuron; arrowheads indicate GABA negative neurons in the MSO. Bars $=20 \mu \mathrm{m}$.

the remainder were negative for GABA immunoreactivity (Fig. 1B). All of the GLY+ and GABA+ perikarya within the LSO neuropil were fusiform or oval in shape, and their size, measured along the major axis of the perikaryon, ranged from 16 to 26 $\mu \mathrm{m}$. The somal shapes of the unlabeled neurons varied from fusiform to polygonal. Roughly one-half of the marginal neurons (neurons that lie immediately within the margins of the LSO, parallel to its transverse curvatures) labeled with GABA antisera; likewise with GLY antisera. Small LSO neurons $(<$ $15 \mu \mathrm{m}$ in length), scattered throughout the medial and middle limbs, were negative for both GABA and GLY immunoreactivity. Examination of the adjacent $1 \mu \mathrm{m}$ sections prepared to assess colocalization of GABA and GLY indicated that most, if not all, of the GABA+ LSO neurons were also weakly GLY+, and vice versa. GABA and GLY immunoreactivity appeared to occur in the same neuronal population (Fig. 2).

Fig. 5. GLY and GABA immunolabeling in the MNTB. A: perisomatic GLY immunoreactive punctate labeling (arrows, for example) is sparse in the MNTB. A, inset: the vast majority of MNTB neurons are intensely GLY immunoreactive (arrow, for example). B: several GABA immunopositive puncta (arrows) can be observed on MNTB neurons. B, inset: while most MNTB neurons are not GABA-immunolabeled (small arrows) a moderately GABA immunoreactive polygonal MNTB neuron is occasionally observed (large arrow). Bars $=20 \mu \mathrm{m}$. 


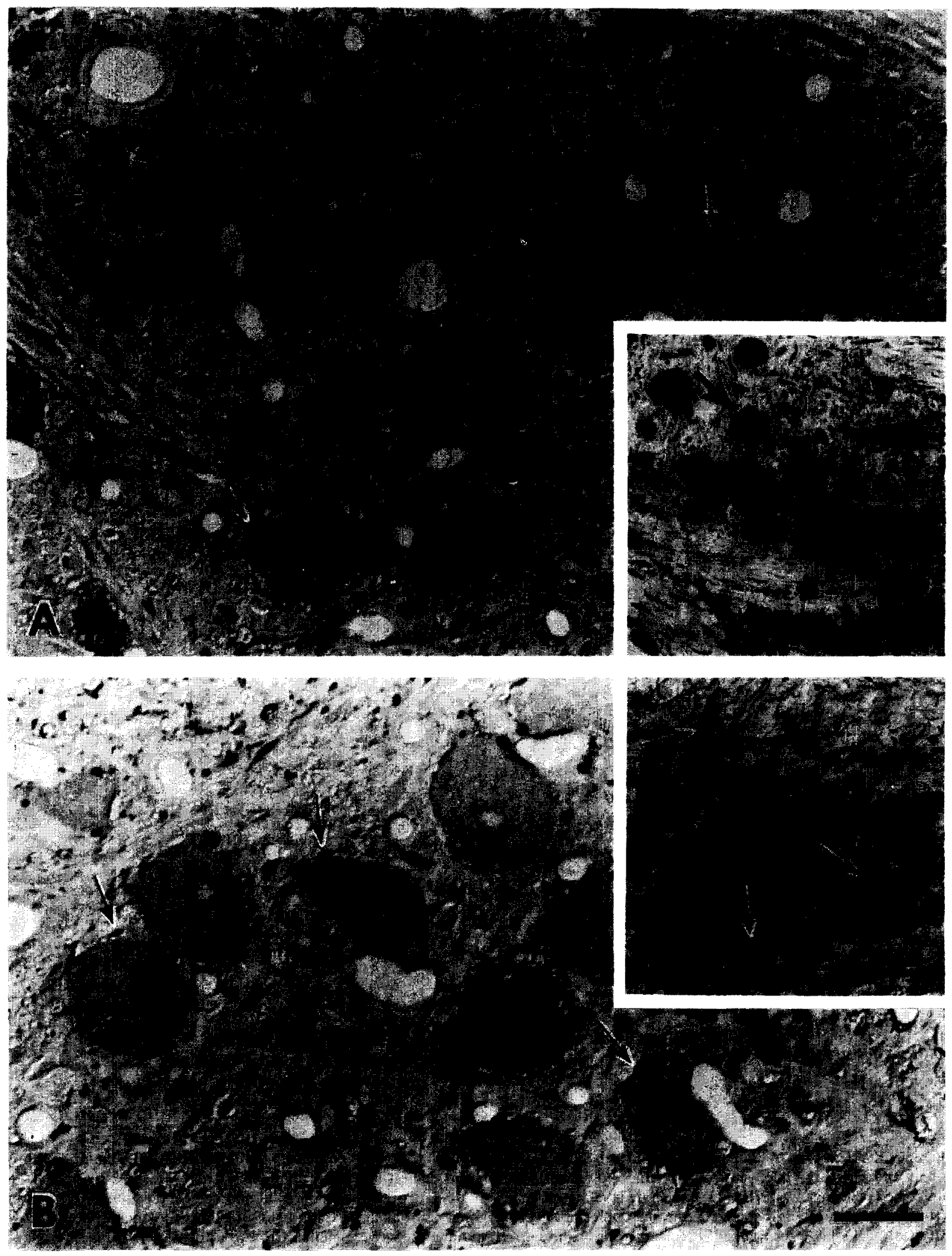



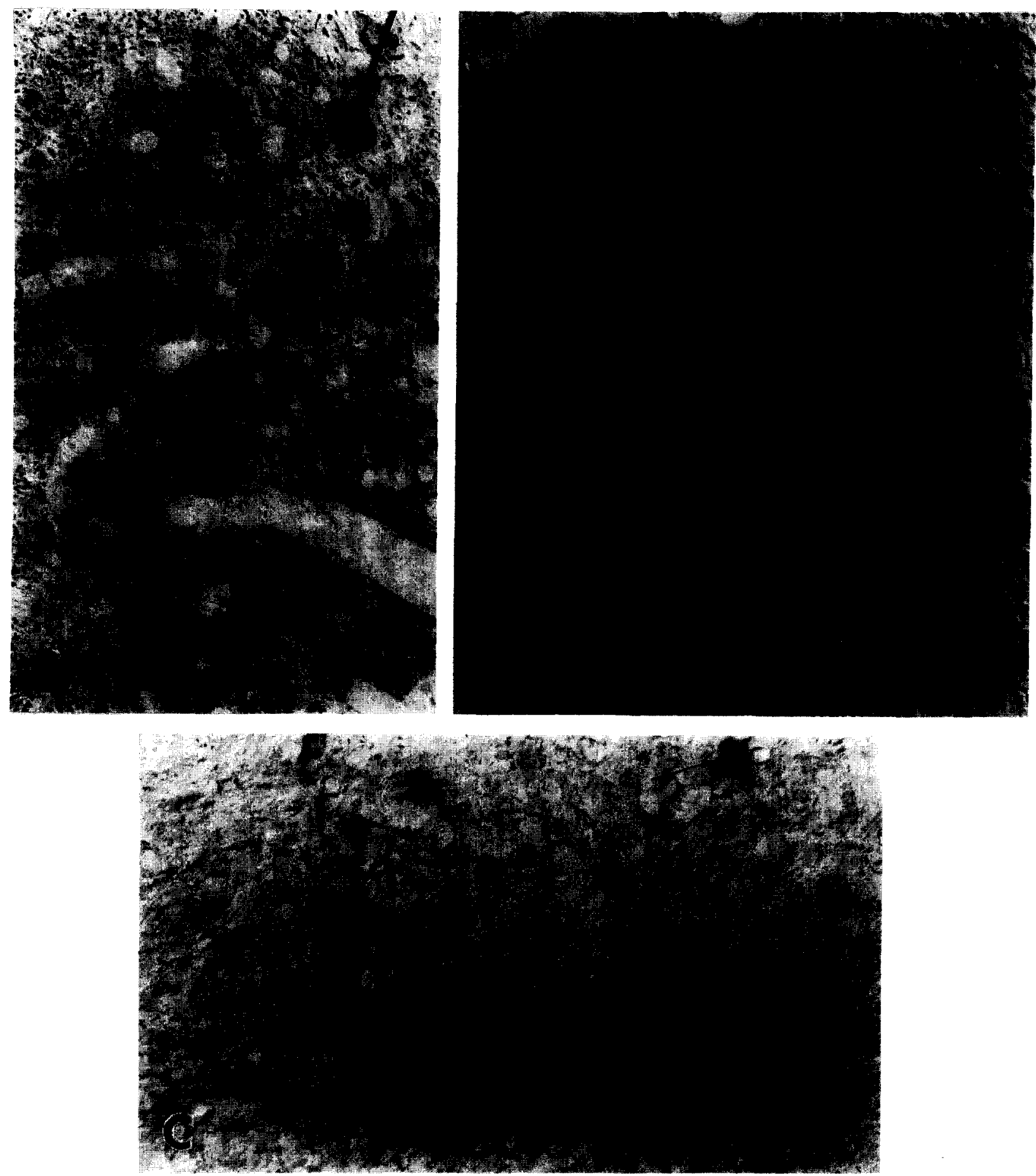

Fig. 6. GLY and GABA immunolabeling in the SPN. A: the neuropil of the SPN contains an abundance of GLY immunopositive fibers and puncta. While a few of the large, polygonal SPN neurons exhibit light GLY immunoreactivity (large arrow), most are unlabeled (short arrows). B: GLY+punctate labeling surrounds the somata of the non-GLY-immunoreactive polygonal SPN neurons (short arrows), while it is sparse on the cell bodies of these polygonal neurons that exhibit light GLY immunolabeling (long arrow). C: most of the large, polygonal SPN neurons are lightly GABA immunoreactive (smaller arrows); a few smaller SPN neurons are more intensely GABA immunostained, as indicated by the larger arrow. Bars $=20 \mu \mathrm{m}$. 
The neuropil of the LSO was rich with intensely GLY+ fibers and puncta (Figs. 1A, 3A). While the profiles of most LSO perikarya were surrounded by $>10 \mathrm{GLY}+$ puncta, most of which appeared to be non-axonal, several of the GLY+ somata were apposed to noticeably fewer of them. The LSO neuropil contained far fewer GABA+ structures (Fig. 3B), many of which appeared to be either dendritic profiles or cross-sections of axons. The cell bodies of the vast majority of the LSO neurons were contacted by only one or two GABA+ puncta.

\section{Medial superior olivary nucleus}

No immunolabeling for GLY was observed in any of the MSO neurons. However, the vast majority of the perikarya of central cell band neurons and marginal neurons were contacted by 5-10 GLY+ puncta (Fig. 4A). A similar density of punctate GLY+labeling was observed on the proximal dendrites of MSO neurons. Coarse, GLY + fibers appeared to pass from the MNTB, through the MSO, en route to the LSO. These fibers may, at least in part, give rise to the puncta seen on the MSO cells.

A very small percentage of MSO neurons were intensely GABA+ (Fig. 4B) and were scattered throughout the rostrocaudal extent of the MSO; the remainder of the cells were unlabeled. Typically, only one GABA-labeled neuron could be identified in a given transverse section through the MSO. They share similar size, shape, and orientation with the majority of the unlabeled MSO neurons in that they are fusiform, $20-25 \mu \mathrm{m}$ in length, with long, tapering dendrites oriented in a horizontal plane. Adjacent GLY-immunostained $1 \mu \mathrm{m}$ sections reveal that GABA+ MSO neurons have patterns of perisomatic and peridendritic GLY punctate labeling similar to those of unlabeled MSO neurons. Like the LSO, the distribution of GABA+ fibers and puncta was sparse; usually 1-4 puncta contacted each neuron.

\section{Medial nucleus of the trapezoid body}

A great majority of the MNTB neurons were intensely GLY +. The shape of these neurons was most often globular, corresponding to the shape of the principal neurons (Fig. 5A, inset). In addition, GLY-labeled neurons were identified with somal shapes similar to those described by Morest ${ }^{66}$ as elongate neurons. Unlabeled cell bodies were only rarely encountered in this nucleus; these were typically polygonal in shape. The MNTB appeared to be the source of the large, GLY+ axons that passed out of the nucleus and coursed around or through the MSO and SPN to approach the LSO and neighboring nuclei. Small numbers (1-5) of GLY+ puncta were observed on the perikaryal surfaces of MNTB neurons (Fig. 5A).

Most neurons in the MNTB were unlabeled by GABA antibodies. Occasionally, a polygonal neuron was observed to be lightly immunolabeled for GABA (Fig. 5B, inset). Of the principal SOC nuclei, the MNTB possessed the most abundant punctiform labeling; 5-10 GABA+ puncta were seen on the perikarya of most MNTB neurons (Fig. 5B).

\section{Superior paraolivary nucleus}

Large, GLY + fibers coursed through the SPN toward the LSO, giving off branches that appeared to ramify to provide the SPN with extensive punctiform GLY labeling (Fig. 6A). Several unlabeled large (25-35 $\mu \mathrm{m})$, polygonal, SPN neurons were surrounded by perisomatic GLY labeling, $>10$ puncta per soma, while lightly GLY+ perikarya of similar size and shape were contacted by $\leqslant 5$ puncta (Fig. 6B). In addition, smaller oval or fusiform neurons (20-25 $\mu \mathrm{m}$ in length) were lightly GLY labeled and were apposed to GLY puncta whose numbers varied from zero to $>10$. Perikaryal immunostaining of the large neurons with GABA antisera was light, while a very small number of 12-20 $\mu \mathrm{m}$ fusiform or oval neurons were moderately GABA+ (Fig. 6C). As in the LSO and MSO, perisomatic and peridendritic labeling with GABA+ puncta was sparse.

\section{Ventral and lateral nuclei of the trapezoid body}

In the VNTB, GLY+ fusiform and polygonal neurons, $20-30 \mu \mathrm{m}$ in length, were identified among unstained neurons of similar shapes and dimensions (Fig. 7A). Smaller (12-15 $\mu \mathrm{m}) \mathrm{GLY}+$ oval neurons were also observed in the VNTB. The LNTB appeared to contain a few subpopulations of GLY+ neurons. The most distinct of these was located ventromedially in the LNTB and contained neurons that were oval or polygonal with sizes ranging from 


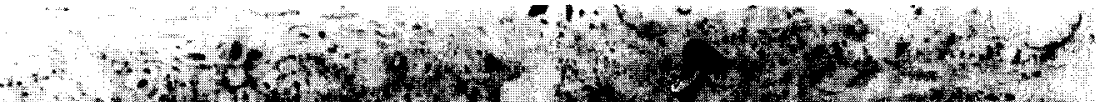

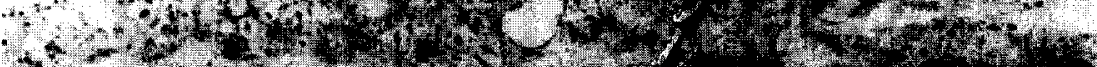

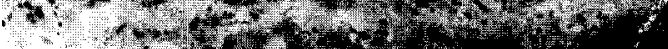

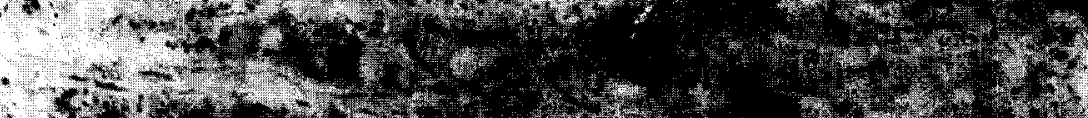

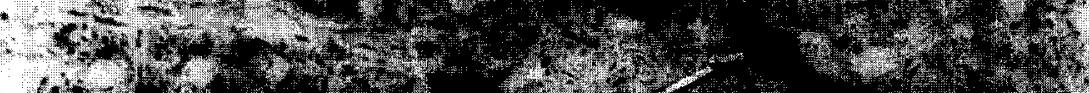

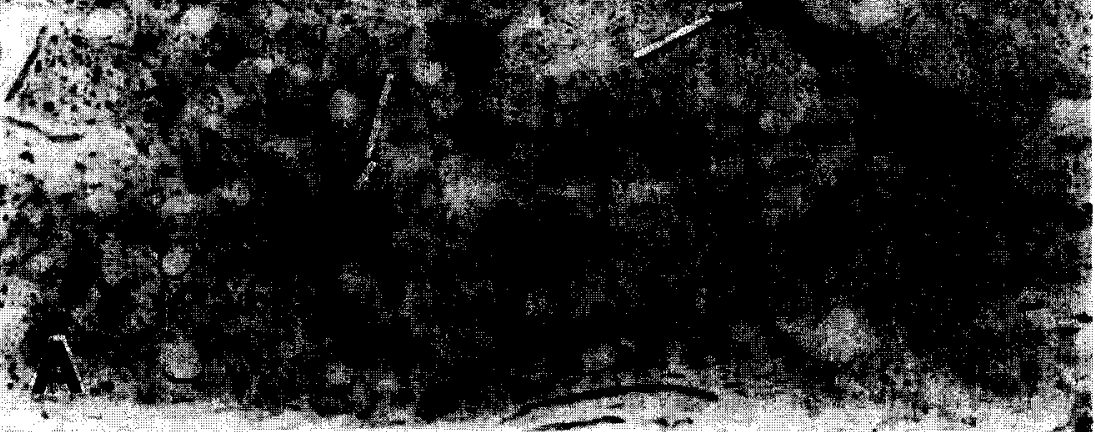

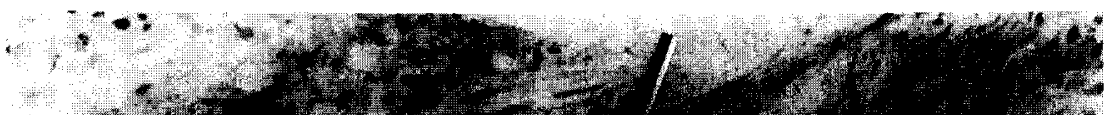

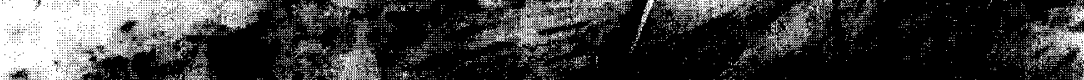

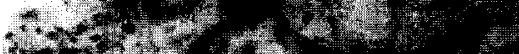
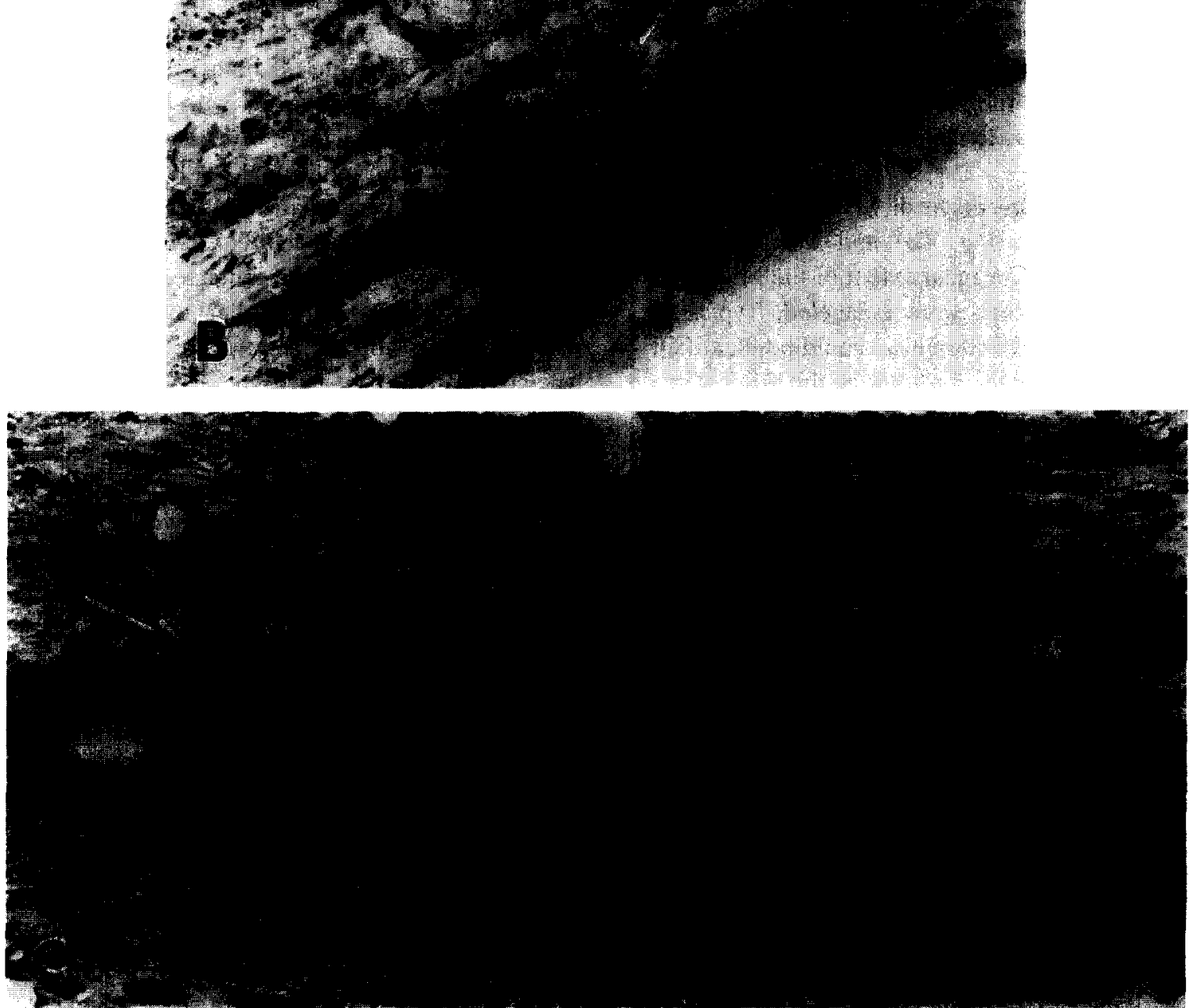

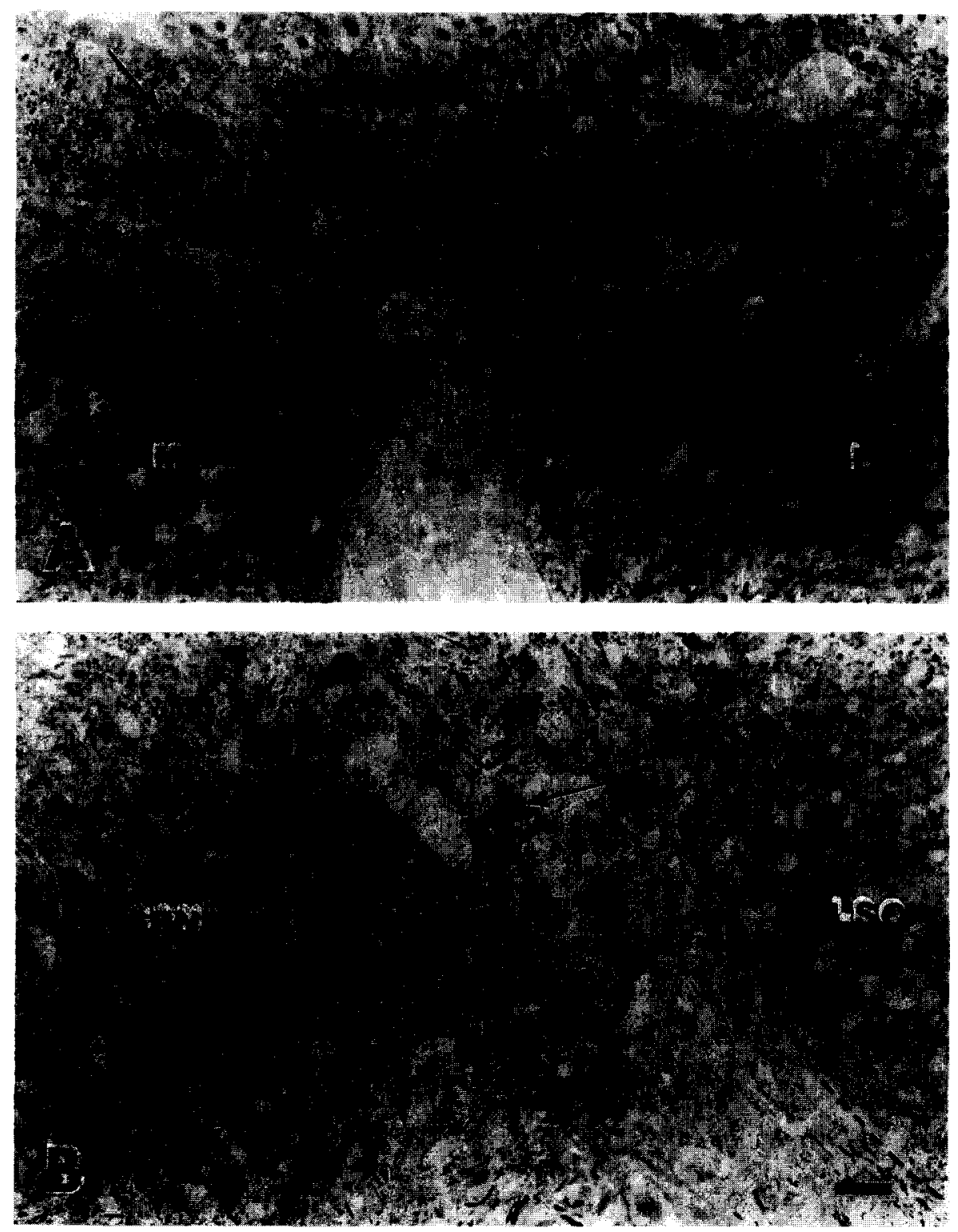

Fig. 8. GLY immunoreactive neurons (arrows) can be identified dorsal to the LSO (A) as well as between the LSO and SPN (B). $\mathrm{M}$, middle limb of the LSO; L, lateral limb of the LSO. Bar $=20 \mu \mathrm{m}$.

16 to $20 \mu \mathrm{m}$ (Fig. 7B). Two other subpopulations were located adjacent to the lateral limb of the LSO, one ventrolaterally, and the other more dorsal, nearer the pole of the lateral limb. The extent of punctate GLY labeling was variable in both the LNTB and VNTB; 0-5 puncta per somal profile was typically observed on both GLY+ and GLY-negative profiles.

In the sections prepared for GABA immunostaining the entire VNTB, as well as the medial portion of the LNTB, characteristically exhibited diffuse background staining. Superimposed on this back-

Fig. 7. GLY immunoreactivity in the VNTB and LNTB, GABA immunolabeling in the VNTB. A: arrows indicate examples of GLY immunoreative VNTB neurons. B: arrow indicates a population of GLY immunopositive neurons located in the medial LNTB. C: arrows indicate areas in the VNTB that contain a great abundance of GABA immunoreactive punctate labeling; stars indicate GABA immunolabeled neurons. Bar $=20 \mu \mathrm{m}$. 
ground were dendritic profiles surrounded by GABA+ puncta (Fig. 7C). The GABA+ punctiform labeling was more abundant in the neuropil of the VNTB than in neuropil anywhere else in the SOC. A majority of the perikarya in both the VNTB and LNTB were moderately GABA-positive, with variable perisomatic labeling of 1-5 GABA+ puncta per labeled or unlabeled somal profile.

Neurons located outside of the 'defined' SOC nuclei

A small number of GLY+ neurons was observed near the dorsal hilus of the LSO (Fig. 8A). They were oval- or fusiform-shaped and $20-25 \mu \mathrm{m}$ in length. There was also a small population of GLY+ cells immediately outside of the medial limb of the LSO, near the dorsal pole of the MSO and the lateral tip of the SPN (Fig. 8B). These two populations fell outside of the boundaries of the named rodent SOC nuclei, but were confined within the superior olivary complex. GLY+ punctate labeling on these neurons was extremely sparse.

\section{DISCUSSION}

The results of the present study, obtained using two different immunoperoxidase immunocytochemical techniques, expand upon the previous findings in the rodent SOC for patterns of immunolabeling with $\mathrm{GLY}^{13,72,102}$, GABA ${ }^{71,91}$, and glutamic acid decarboxylase (GAD), the synthesizing enzyme for $\mathrm{GABA}^{64,76}$. However, there is some disparity between the patterns of GLY and GABA immunolabeling observed in the rodent LSO and those recently described in the cat ${ }^{81}$. While the results of the present study augment our knowledge regarding the presence of GABA and GLY in the SOC, they also raise several questions that pertain mostly to the function of these inhibitory neurontransmitters in circuits involving the periolivary nuclei.

\section{Lateral superior olive}

A major role for inhibition has been defined in the LSO, a nucleus that is a primary site for the convergence of binaural input. The majority of its neurons are excited by ipsilateral sound stimuli and are inhibited by the simultaneous presentation of a contralateral tone of similar frequency ${ }^{19,21,37,46,93}$. These ipsi-excitatory and contra-inhibitory (EI) units are well suited to function as comparators of interaural intensity differences ${ }^{18}$ and as such may be employed to localize sounds.

Several lines of evidence support the contention that GLY is the neurotransmitter mediating contralaterally-induced inhibition in the LSO. In the chinchilla, iontophoretic application of GLY to LSO neurons during an ipsilateral tone presentation mimics binaural inhibition, while the simultaneous application of the GLY antagonist, strychnine, abolishes this effect ${ }^{65}$. High affinity uptake of tritiated GLY by synaptic terminals has been observed in the cat $\mathrm{LSO}^{84}$. The somata of most LSO neurons are surrounded by GLY-immunoreactive puncta as observed in this study and by others using guinea pigs $^{102}$ and cats $^{81}$. This GLY labeling appears to be primarily in presynaptic terminals. Ultrastructural studies indicate that a large percentage of LSO perikarya appose numerous flat vesicle terminals in the cat $^{22,51}$ and gerbil ${ }^{52}$, while their dendrites contact large, round vesicle terminals in cats ${ }^{22}$ and rats ${ }^{104}$. In the cochlear nuclei and superior olive, synapses with flat vesicle terminals appear to contain the inhibitory transmitter, GLY ${ }^{9.54}$. The large, round vesicle terminals in the LSO possibly contain an excitant amino acid neurotransmitter. Using colloidal gold immunoelectron microscopy, we have recently observed intense glutamate immunoreactivity associated with this synaptic type ${ }^{49}$. This finding would be consistent with the evidence in the ventral cochlear nucleus that excitant amino acids may be associated with spherical cells $8,12,61$. The ipsilateral afferents to the LSO are supplied primarily by the small spherical cell region of the anteroventral cochlear nucleus ${ }^{24,88}$, 97,99 , and may be a major source of the large, round vesicle axodendritic synapses in the $\mathrm{LSO}^{24}$.

The majority of guinea pig LSO projection neurons are $\mathrm{GABA}+$. This observation is supported by previous investigations in guinea pig and gerbil using GABA and GAD antibodies ${ }^{76,91}$. Moore and Moore $^{64}$ also identified GAD + neurons in the rat, but they were typically smaller than the principal neurons and therefore were categorized as small neurons. In the guinea pig, GABA+ LSO neurons also appear to co-contain GLY. While this may also be the case in the cat, major differences do exist. Saint Marie et al ${ }^{81}$ found a very small percentage $(<$ $3 \%$ ) of cat LSO projection neurons to be GABA+; 
and while they did co-label with GLY, a much larger population of GLY+ LSO neurons did not co-label with GABA. In addition, at least two-thirds of the guinea pig LSO neurons appear to be GABA+ and/or GLY + while only $\sim 40 \%$ of the cat LSO neurons are such ${ }^{81}$. These discrepancies between the cat and rodent LSO in their GABA and GLY immunocytochemistry lack an immediate explanation beyond the fact that they may reflect interspecies differences. LSO projection neurons send axons rostrally and bilaterally to the dorsal nucleus of the

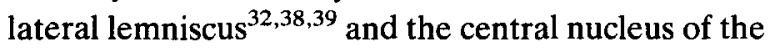
inferior colliculus ${ }^{1,20,68,79,88}$. Because most of the guinea pig LSO neurons are likely to be GABAergic they, along with the GAD+ neurons of the dorsal nucleus of the lateral lemniscus ${ }^{3,64,76}$, would contribute significantly to the extensive GABA+ punctate labeling observed in the central nucleus of the inferior colliculus ${ }^{64,76}$. It remains to be seen whether or not the rodent LSO sends facilitatory output to the contralateral inferior colliculus and mixed facilitatory and inhibitory projections ipsilaterally as proposed in the cat $^{81}$.

The density and distribution of GABA+ puncta in the LSO can be described as light and scattered. It should be stated here that this is also the case in most other SOC nuclei. The pattern of GABA punctate labeling has been examined in the guinea pig SOC using immuno-electron microscopy ${ }^{54}$. GABA + terminals have a limited distribution in the LSO, MSO, MNTB and SPN. These terminals can be distinguished from GLY+ ones in that they are typically smaller, contain pleomorphic oval vesicles with scattered dense core vesicles, and possess broader active zones than those found in GLY+ terminals. GABA synapses of similar morphology have also been described in the guinea pig cochlear nucleus $^{10,69}$. Several potential sources of GABA input to the SOC exist in the auditory brainstem since GABA+ and GAD+ neurons have been identified in nuclear complexes known to project to the rodent SOC: the ventral cochlear nucleus ${ }^{76,91,101}$, the inferior colliculus ${ }^{64,76,91}$, the nuclei of the lateral lemniscus ${ }^{3,64,76}$, as well as the SOC itself ${ }^{64,72,91}$. The role GABA plays in the processing of binaural input or in any other function is unclear because the source providing GABA input to morphologically or functionally distinct entities in the SOC has not been positively identified. Elucidation of the GABAergic pathways and/or loops affiliated with the SOC will be necessary to fully understand the functional significance of GABAergic input to this area.

\section{Medial nucleus of the trapezoid body}

The vast majority of guinea pig MNTB neurons are GLY + . These cells correspond to the principal neurons and possibly to the elongate neurons described in the cat by Morest ${ }^{66}$. MNTB cells are contacted by small numbers of GLY immunoreactive puncta. It is not known whether these puncta originate from recurrent collaterals arising within the MNTB itself, from elsewhere in the SOC or, perhaps, from the cochlear nucleus.

The MNTB receives excitatory input from the globular cell region of the contralateral ventral cochlear nucleus ${ }^{47,94,98}$ and in turn projects ipsilaterally to the LSO, MSO, SPN, LNTB, and the nuclei of the lateral lemniscus ${ }^{17,66,86,109}$. Based on the immunocytochemical findings of this study and others ${ }^{13,72,102}$, these projections that represent input from the contralateral ear are probably glycinergic, and thus inhibitory. Taking into account the projection of MNTB to $\mathrm{LSO}^{17,40,74,86,109}$, lesioning the guinea pig MNTB with the excitotoxin, kainic acid, produces a subsequent loss of perisomatic GLY immunoreactive labeling in the $\mathrm{LSO}^{16}$, where, in the chinchilla, the iontophoretic application of glycine is known to inhibit the activity of most of its neurons ${ }^{65}$.

\section{Medial superior olive}

The MSO plays a role in low frequency sound localization by detecting interaural time or phase differences ${ }^{37,41,42,46,67,107}$. Most MSO neurons are configured so that their lateral dendrites receive input from the ipsilateral anteroventral cochlear nucleus (AVCN) while their medial dendrites are contacted by terminals from the contralateral $\mathrm{AVCN}^{80,88,98}$. This binaural input is excitatory and evokes maximal facilitation of MSO units when the input to both ears arrives exactly in phase ${ }^{41,42,46}$. However, many MSO neurons are inhibited when, at higher sound intensities, the binaural inputs arrive $180^{\circ}$ out of phase ${ }^{41,42,46}$. For over one-half of the MSO units tested, the inhibitory responses can be elicited by both ipsilateral and contralateral sound stimuli $^{106,108}$. 
Inhibition in the MSO could be mediated by glycine. Pleomorphic (oval or flat) vesicle synaptic terminals similar to the inhibitory ones observed on the LSO perikarya have been observed on the soma and proximal dendrites of MSO neurons ${ }^{57,60,70}$. In the guinea pig, most of the flat vesicle terminals have been demonstrated to be GLY $+{ }^{54}$, and their distribution parallels the perisomatic punctate GLY labeling on most MSO neurons observed in this study. No obvious differences exist between marginal neurons and central cell band neurons in the guinea pig with regard to relative numbers and distribution of GLY + puncta. A species-related difference may be found in the cat, where central cell band neurons appose few terminals exhibiting high affinity GLY uptake $^{82,83}$ when compared to marginal neurons. There are two probable sources of GLY input to the MSO from within the SOC. First, a small number of axon collaterals from the MNTB appear to terminate on MSO neurons in guinea pigs ${ }^{17}$ and cats ${ }^{83,86}$. These fibers may be glycinergic if they originate from MNTB principal or elongate neurons; if so, they would represent a source of contralateral inhibition to the MSO. Second, the LNTB projects to the ipsilateral $\mathrm{MSO}^{23}$. This nucleus contains GLY+ neurons as observed in this study and by Peyret et al. ${ }^{72}$. Most GLY + LNTB neurons appear also to contain GABA and it is not certain what effects co-containment of these compounds would have on the labeling characteristics of the LNTB-MSO projection. Nevertheless, the possibility remains that the LNTB is a potential source of ipsilateral GLY. mediated inhibitory input to the MSO.

The vast majority of MSO neurons project ipsilaterally to the inferior colliculus $1,20,32,55,79,88$ and the nuclei of the lateral lemniscus ${ }^{38,55}$. Because the vast majority of MSO neurons are not labeled with GABA or GLY, it is conceivable then that this projection could be facilitatory. The very small numbers of GABA + neurons observed in the MSO may serve, at least in part, either as interneurons providing other MSO neurons with GABAergic input, or they may possibly project outside to an as yet unidentified auditory nucleus. It is also conceivable that these GABA+ neurons may correspond to the equally small number of MSO neurons observed to project to the contralateral inferior colliculus in the $\mathrm{cat}^{20}$ and gerbil ${ }^{68}$.

\section{The periolivary nuclei}

The remaining subnuclei of the SOC together compose the PON. These nuclei contain heterogeneous populations of neurons of potentially great structural, physiological, and biochemical diversity. The potential for the PON to possess an abundant variety of neurotransmitters or neuromodulators has been suggested in the cat $\mathrm{VNTB}^{4}$. Neurons of the PON receive input from and, in turn, project to virtually every major brainstem auditory center. They receive ascending input from the cochlear nucleus ${ }^{6,97}$ descending projections from the inferior colliculus $^{11,33}$ and lemniscal nuclei ${ }^{58,105}$ as well as input from within the $\mathrm{SOC}^{17,66,86,109}$. The PON project to the cochlea (for a review of the literature, see Warr et al ${ }^{100}$ ), cochlear nuclei ${ }^{2,6,31,87}$, principal nuclei of the $\mathrm{SOC}^{40}$, nuclei of the lateral lemniscus ${ }^{38}$, and inferior colliculus ${ }^{1,2,15,20,79}$.

To date, little is known about the functional significance of inhibition associated with the PON. Few inferences can be made without knowledge of the connections to and projections from the GABA+ and GLY+ elements. GABA+ efferent fibers and terminals have been identified beneath both inner and outer hair cells $s^{34,36,92}$. In addition, selective high affinity uptake of tritiated GABA and one of its analogues, nipecotic acid, by efferent endings beneath outer and inner hair cells has been observed in gerbils ${ }^{80,85}$. The nipecotic acid-labeled efferents were traced back to cells of origin in the VNTB, LNTB, and between MSO and MNTB ${ }^{80}$. In the guinea pig as well, these nuclei contain olivocochlear neurons $^{77}$ and are also areas where the present study shows that GABA+ neurons exist. GLY, on the other hand, does not appear to be a major neurotransmitter candidate in the olivocochlear system ${ }^{35,85}$.

The guinea pig SPN receives major glycinergic input that originates mostly from the MNTB. Anterograde tracing studies using the plant lectin Phaseolus vulgaris-leucoagglutinin demonstrate an extensive topographical projection from the MNTB to the $\mathrm{SPN}^{17}$. These studies also indicate a smaller projection from MNTB to the LNTB that may be glycinergic. Known projections from the guinea pig SPN probably include those of medial olivocochlear neurons ${ }^{77}$ as well as axons that ascend to the dorsal nucleus of the lateral lemniscus ${ }^{50}$. 
Neurons from all of the cat periolivary nuclei project to the cochlear nucleus ${ }^{2,31,87}$; this may also be the case for the guinea pig. Most of these neurons are probably either GABAergic or glycinergic, and their projections could account for a large portion of the widespread GABA and GLY punctate labeling identified in all divisions of the cat and rodent cochlear nucleus $5,13,64,76,91,102$.

Reciprocal connections have been identified between the PON and the central nucleus of the inferior colliculus in the cat ${ }^{11}$. Because of the extensive GABA+ and GAD+ somatic and punctate labeling in these nuclei ${ }^{64,76,91}$, it would be plausible to assume that the reciprocal connections may, in part, be GABAergic. However, it should be noted that such reciprocity has not been observed in the rat; its colliculo-olivary fibers apparently do not project to the PON neurons that, in turn, send axons to the inferior colliculus ${ }^{33}$.

Further knowledge of the roles GABA and GLY play in the PON will rely upon a thorough understanding of its connections. The acquisition of this knowledge will be challenging because, often, individual neurons belonging to a population of interest

\section{REFERENCES}

1 Adams, J.C., Ascending projections of the inferior colliculus, J. Comp. Neurol., 183 (1979) 519-538.

2 Adams, J.C., Cytology of periolivary cells and the organization of their projections in the cat, J. Comp. Neurol., 215 (1983) 275-289.

3 Adams, J.C. and Mugnaini, E., Dorsal nucleus of the lateral lemniscus: a nucleus of GABAergic projection neurons, Brain Res. Bull., 13 (1984) 585-590.

4 Adams, J.C. and Mugnaini, E., Patterns of immunostaining with antisera to peptides in the auditory brainstem of cat, Soc. Neurosci. Abstr., 11 (1987) 32.

5 Adams, J.C. and Mugnaini, E., Patterns of glutamate decarboxylase immunostaining in the feline cochlear nuclear complex studied with silver enhancement and electron microscopy, J. Comp. Neurol., 262 (1987) 375401.

6 Adams, J.C. and Warr, W.B., Origins of axons in the cat's acoustic striae determined by injection of horseradish peroxidase into severed tracts, J. Comp. Neurol., 170 (1976) 107-121.

7 Aitkin, L.M., Webster, W.R., Veale, J.L. and Crosby, D.C., Inferior colliculi. I. Comparison of response properties of neurons in central, pericentral, and external nuclei of aduit cat, J. Neurophysiol., 38 (1975) 1196-1207.

8 Altschuler, R.A., Neises, G.R., Harmison, G.G., Wenthold, R.J. and Fex, J., Immunocytochemical localization of aspartate aminotransferase immunoreactivity in the cochlear nucleus of the guinea pig, Proc. Natl. Acad. Sci. within the PON will not be compartmentalized and will, instead, be scattered among neurons of populations that differ from each other in function, connections, and chemistry. Future studies will need to focus on combining techniques such as immunocytochemistry with tract-tracing methods in order to accurately delineate the neurochemistry of individual pathways to and from the PON. Of course, this would apply to the other components of the SOC as well.

\section{ACKNOWLEDGEMENTS}

We are most grateful to Ms. Snigdha Singh and Ms. Rebecca L. Szczygiel for their excellent technical assistance, to Ms. Pamela A. Helfert and Mrs. Mary L. Stuckmeyer for the adept preparation of this manuscript, and to Drs. Sanford C. Bledsoe, Jr. and Susan E. Shore for their thoughtful reviews of the manuscript. This research was supported by National Institutes of Health Grants NS24369 and NS07106, as well as by a grant from the Deafness Research Foundation. A preliminary report on some of these findings has appeared ${ }^{53}$.

U.S.A., 78 (1981) 6553-6557.

9 Altschuler, R.A., Betz, H., Parakkal, M.H., Reeks, K.A. and Wenthold, R.J., Identification of glycinergic synapses in the cochlear nucleus through immunocytochemical localization of the postsynaptic receptor, Brain Research, 369 (1986) 316-320.

10 Altschuler, R.A., Hoffman, D.W. and Wenthold, R.J., Neurotransmitters of the cochlea and cochlear nucleus: immunocytochemical evidence, Am. J. Otolaryngol., 7 (1986) 100-106.

11 Anderson, R.A., Roth, G.L., Aitkin, L.M. and Merzenich, M.M., The efferent projections of the central nucleus and the pericentral nucleus of the inferior colliculus in the cat, J. Comp. Neurol., 194 (1980) 649-662.

12 Aoki, E., Semba, R., Kato, K. and Kashiwamata, S., Purification of specific antibody against aspartate and immunocytochemical localization of aspartergic neurons in the rat brain, Neuroscience, 21 (1987) 755-765.

13 Aoki, E., Semba, R., Keino, J., Kato, K. and Kashiwamata, S., Glycine-like immunoreactivity in the rat auditory pathway, Brain Research, 442 (1988) 63-71.

14 Aprison, J.H., Daly, E.C., Shank, R.P. and McBride, W.J., Neurochemical evidence for glycine as a transmitter and a model for its intrasynaptosomal compartmentation. In S. Berl, D.D. Clarke and D. Schneider (Eds.), Metabolic Compartmentation of Neurotransmission, Raven, New York, 1975, pp. 37-63.

15 Beyerl, B.D., Afferent projections to the central nucleus of the inferior colliculus in the rat, Brain Research, 145 
(1978) 209-223.

16 Bledsoe, S.C., Altschuler, R.A., Wenthold, R.J. and Prasad, V., Immunocytochemical localization of glycine in the guinea pig superior olivary complex: lesion studies, Assoc. Res. Otolaryngol. Midwinter Meeting Abstr., 10 (1987) 157.

17 Bledsoe, S.C., Pandya, P., Altschuler, R.A. and Helfert, R.H., Axonal projections of PHA-L-labeled neurons in the medial nucleus of the trapezoid body, Soc. Neurosci. Abstr., 14 (1988) 491.

18 Boudreau, J.C. and Tsuchitani, C., Cat superior olive S-segment cell discharge to tonal stimulation, Contrib. Sensory Phsyiol., 4 (1970) 143-213.

19 Brownell, W.E., Manis, P.B. and Ritz, L.A., Ipsilateral inhibitory responses in the cat lateral superior olive S-segment, Brain Research, 177 (1979) 189-193.

20 Brunso-Bechtold, J.K., Thompson, G.C. and Masterton, R.B., HRP study of the organization of auditory afferents ascending to central nucleus of inferior colliculus in cat, $J$. Comp. Neurol., 197 (1981) 705-722.

21 Caird, D. and Klinke, R., Processing of binaural stimuli by cat superior olivary complex neurons, Exp. Brain Res., 52 (1983) 385-399.

22 Cant, N.B., The fine structure of the lateral superior olivary nucleus of the cat, J. Comp. Neurol., 227 (1984) 63-77.

23 Cant, N.B., Projections to lateral and medial superior olivary nuclei from the spherical and globular bushy cells of the AVCN. In R.A. Altschuler, R.P. Bobbin, D.W. Hoffman and B.M. Clopton (Eds.), Neurobiology of Hearing: the Central Auditory System, Raven, New York, in press.

24 Cant, N.B. and Casseday, J.H., Projections from the anteroventral cochlear nucleus to the lateral and medial superior olivary nuclei, J. Comp. Neurol., 247 (1986) 457-476.

25 Canzek, V. and Reubi, J.C., The effect of cochlear nerve lesion on the release of glutamate, aspartate, and GABA from cat cochlear nucleus, in vitro, Exp. Brain Res., 38 (1980) 437-441.

26 Caspary, D.M., Havey, D.C. and Faingold, C.L., Effects of microiontophoretically applied glycine and GABA on neuronal response patterns in the cochlear nuclei, Brain Research, 172 (1979) 179-185.

27 Comis, S.D., Centrifugal inhibitory processes affecting neurons in the cat cochlear nucleus, $J$. Physiol. (Lond.), 210 (1970) 751-760.

28 Comis, S.D. and Whitfield, I.C., Influence of centrifugal pathways on unit activity in the cochlear nucleus, $J$. Neurophysiol., 31 (1968) 62-68.

29 Curtis, D.R. and Johnston, G.A.R., Amino acid neurotransmitters in the mammalian central nervous system, Rev. Physiol., 69 (1974) 98-188.

30 Davies, W.E., The distribution of GABA transaminasecontaining neurons in the cat cochlear nucleus, Brain Research, 83 (1975) 27-33.

31 Elverland, H.H., Descending connections between the superior olive and cochlear nuclear complexes in the cat studied by autoradiographic and horseradish peroxidase methods, Exp. Brain Res., 27 (1977) 397-412.

32 Elverland, H.H., Ascending and intrinsic projections of the superior olivary complex in the cat, Exp. Brain Res., 32 (1978) 117-134.

33 Faye-Lund, H., Projection from the inferior colliculus to the superior olivary complex in the albino rat, Anat. Embryol., 175 (1986) 35-52.

34 Fex, J. and Altschuler, R.A., Glutamic acid decarboxylase immunoreactivity of olivocochlear neurons in the organ of Corti of guinea pig and rat, Hearing Res., 15 (1984) 123-131.

$35 \mathrm{Fex}$, J. and Altschuler, R.A., Unpublished observations.

36 Fex, J., Altschuler, R.A., Kachar, B., Wenthold, R.J. and Zempel, J.M., GABA visualized by immunocytochemistry in the guinea pig cochlea in axons and endings of efferent neurons, Brain Research, 366 (1986) 100-117.

37 Galambos, R., Schwartzkopff, J. and Rupert, A., Microelectrode study of superior olivary nuclei, Am. J. Physiol., 197 (1959) 527-536.

38 Glendenning, K.K., Brunso-Bechtold, J.K., Thompson, G.C. and Masterton, R.B., Ascending auditory afferents to the nuclei of the lateral lemniscus, J. Comp. Neurol., 197 (1981) 673-703.

39 Glendenning, K.K. and Masterton, R.B., Acoustic chiasm: efferent projections of the lateral superior olive, J. Neurosci., 3 (1983) 1521-1537.

40 Glendenning, K.K., Hutson, K.A., Nudo, R.J. and Masterton, R.B., Acoustic chiasm. II. Anatomical basis of binaurality in lateral superior olive of cat, J. Comp. Neurol., 232 (1985) 261-285.

41 Goldberg, J.M. and Brown, P.B., Functional organization of the dog superior olivary complex: an anatomical and electrophysiological study, $J$. Neurophysiol., 31 (1968) 639-656.

42 Goldberg, J.M. and Brown, P.B., Response of binaural neurons of dog superior olivary complex to dichotic tonal stimuli: some physiological mechanisms of sound localization, J. Neurophysiol., 32 (1969) 613-636.

43 Godfrey, D.A., Carter, J.A., Berger, S.J., Lowry, O.H. and Matschinsky, F.M., Quantitative histochemical mapping of candidate transmitter amino acids in cat cochlear nucleus, J. Histochem. Cytochem., 25 (1977) 417-431.

44 Godfrey, D.A., Carter, J.A., Lowry, O.H. and Matschinsky, F.M., Distribution of gamma-aminobutyric acid, glycine, glutamate and aspartate in the coclear nucleus of the rat, J. Histochem. Cytochem., 26 (1978) 118-126.

45 Guinan, J.J., Guinan, S.S. and Norris, B.E., Single auditory units in the superior olivary complex. I. Responses to sounds and classifications based on physiological properties, Int. J. Neurosci., 4 (1972) 101-120.

46 Guinan, J.J., Guinan, S.S. and Norris, B.E., Single auditory units in the superior olivary complex. II. Locations of unit categories and tonotopic organization, Int. J. Neurosci., 4 (1972) 147-166.

47 Harrison, J.M. and Warr, W.B., The cochlear nucleus and ascending pathways of the medulla, J. Comp. Neurol., 119 (1962) 341-380.

48 Held, H., Die zentrale Gehorleitung, Arch. Anat. Physiol. Anat. Abt., (1893) 201-248.

49 Helfert, R.H. and Altschuler, R.A., unpublished observations.

50 Helfert, R.H. and Bledsoe Jr., S.C., unpublished observations.

51 Helfert, R.H. and Schwartz, I.R., Morphological evidence for the existence of multiple neuronal classes in the cat lateral superior olivary nucleus, J. Comp. Neurol., 244 (1986) 533-549.

52 Helfert, R.H. and Schwartz, I.R., Morphological features of five neuronal classes in the gerbil lateral superior 
olivary nucleus, Am. J. Anat., 179 (1987) 55-69.

53 Helfert, R.H., Altschuler, R.A. and Wenthold, R.J., GABA and glycine immunoreactivity in the guinea pig superior olivary complex, Soc. Neurosci. Abstr., 13 (1987) 544.

54 Helfert, R.H., Bonneau, J.M., Wenthold, R.J. and Altschuler, R.A., Distribution of GABA and glycine immunoreactive synapses in the guinea pig superior olivary complex, Soc. Neurosci. Abstr., 14 (1988) 487.

55 Henkel, C.S. and Spangler, K.M., Organization of the efferent projections of the medial superior olivary nucleus in the cat as revealed by HRP and autoradiographic tracing methods, J. Comp. Neurol., 221 (1983) 416-428.

$56 \mathrm{Hsu}$, S.-M., Raine, L. and Fanger, H., Use of avidinbiotin-peroxidase complex $(A B C)$ in immunoperoxidase techniques: a comparison between $\mathrm{ABC}$ and unlabeled antibody (PAP) procedures, J. Histochem. Cytochem., 29 (1981) 577-580.

57 Kiss, A. and Majorossy, K., Neuron morphology and synaptic architecture in the medial superior olivary nucleus, Exp. Brain Res., 52 (1983) 315-327.

$58 \mathrm{Kudo}, \mathrm{M}$., Projections of the nuclei of the lateral lemniscus in the cat: an autoradiographic study, Brain Research, 221 (1981) 57-69.

59 Lavilla, I., Algunos detalles concierneintes a la oliva superio y focos accessorios, Rev. Trimestr. Micrograf., 3 (1898) $75-83$.

60 Lindsay, B.G., Fine structure and distribution of axon terminals from the cochlear nucleus on neurons in the medial superior olivary nucleus of the cat, J. Comp. Neurol., 160 (1975) 81-105.

61 Madl, J.E., Larson, A.A. and Beitz, A.J., Monoclonal antibody specific for carbodiimide-fixed glutamate: immunocytochemical localization in the rat, J. Histochem. Cytochem., 34 (1986) 317-326.

62 Masterton, R.B., Jane, J.A. and Diamond, I.T., Role of brainstem auditory structures in sound localization. I. Trapezoid body, superior olive and lateral lemniscus, $J$. Neurophysiol., 30 (1967) 341-359.

63 Masteron, R.B., Thompson, G.C., Brunso-Bechtold,, J.K. and RoBards, M.J., Neuroanatomical basis of binaural phase difference analysis for sound localization: a comparative study, J. Comp. Physiol Psychol., 89 (1975) 379-386.

64 Moore, J.K. and Moore, R.Y., Glutamic acid decarboxylase-like immunoreactivity in brainstem auditory nuclei of the rat, J. Comp. Neurol., 260 (1987) 157-174.

65 Moore, M.J. and Caspary, D.M., Strychnine blocks binaural inhibition in lateral superior olivary neurons, $J$. Neurosci., 3 (1983) 237-242.

66 Morest, D.K., The collateral system of the medial nucleus of the trapezoid body of the cat, its neuronal architecture and relation to the olivo-cochlear bundle, Brain Research, 9 (1968) 288-311.

67 Moushigian, G., Rupert, A.L. and Whitcomb, M.A., Brainstem neuronal response patterns to monaural and binaural tones, J. Neurosphysiol., 27 (1964) 1174-1191.

68 Nordeen, K.W., Killackey, H.P. and Kitzes, L.M., Ascending auditory projections to the inferior colliculus in the adult gerbil, Meriones unguiculatus, J. Comp. Neurol., 214 (1983) 131-143.

69 Oberdorfer, M.D., Parakkal, M.J., Altschuler, R.A. and Wenthold, R.J., Ultrastructural localization of GABAimmunoreactive terminals in the anteroventral cochlear nucleus of the guinea pig, Hearing Res., 33 (1988) 229-238.

70 Perkins, R.E., An electron microscopic study of synaptic organization in the medial superior olive of normal and experimental chinchillas, J. Comp. Neurol., 148 (1973) 387-416.

71 Peyret, D., Geffard, M. and Aran, J.-M., GABA immunoreactivity in the primary nuclei of the auditory central nervous system, Hearing Res., 23 (1986) 115-121.

72 Peyret, D., Campistron, G., Geffard, M. and Aran, J.-M., Glycine immunoreactivity in the brainstem auditory and vestibular nuclei of the guinea pig, Acta Otolaryngol., 104 (1987) 71-76.

73 Ramón y Cajal, S., Histologie du Système Nerveux de l'Homme et des Vertébrés, Vol. I, Inst. Ramón y Cajal, Madrid, 1909 (1952 reprint).

74 Rasmussen, G.L., The olivary peduncle and other fiber connections of the superior olivary complex, J. Comp. Neurol., 84 (1946) 141-219.

75 Roberts, E. and Kuriyama, K., Biochemical-physiological correlations in studies of the $\gamma$-aminobutyric acid system, Brain Research, 8 (1968) 1-35.

76 Roberts, R.C. and Ribak, C.E., GABAergic neurons and axon terminals in the brainstem auditory nuclei of the gerbil, J. Comp. Neurol., 258 (1987) 267-280.

77 Robertson, D., Brainstem location of efferent neurons projecting to the guinea pig cochlea, Hearing Res., 20 (1985) 79-84.

78 Rosenweig, M.R. and Amon, A.H., Binaural interaction in the medulla of the cat, Experientia, 11 (1955) 498-499.

79 Roth, G.L., Aitkin, L.M., Andersen, R.A. and Merzenich, M.M., Some features of the spatial organization of the central nucleus of the inferior colliculus of the cat, $J$. Comp. Neurol., 182 (1978) 661-680.

80 Ryan, A.F. and Schwartz, I.R., Nipecotic acid: preferential accumulation in the cochlea by GABA uptake systems and selective retrograde transport to the brainstem, Brain Research, 399 (1986) 299-403.

81 Saint Marie, R.L., Ostapoff, E.-M., Morest, D.K. and Wenthold, R.J., A glycine-immunoreactive projection of the cat lateral superior olive: possible role in midbrain ear dominance, J. Comp. Neurol., 279 (1989) 382-396.

82 Schwartz, I.R., Differential tritiated amino acid labeling of synaptic terminals in the cat medial superior olivary nucleus, Assoc. Res. Otolaryngol. Midwinter Meeting Abstr., 5 (1982) 21.

83 Schwartz, I.R., Axonal organization in the cat medial superior olive, Cont. Sensory Physiol., 8 (1984) 99-129.

84 Schwartz, I.R., Autoradiographic studies of amino acid labeling of neural elements in the auditory brainstem. In D.G. Drescher (Ed.), Auditory Biochemistry, Thomas, Springfield, IL, 1985, pp. 258-277.

85 Schwartz, I.R. and Ryan, A.F., Amino acid labeling patterns in the efferent innervation of the cochlea: an electron microscopic autoradiographic study, J. Comp. Neurol., 246 (1986) 500-512.

86 Spangler, K.M., Warr, W.B. and Henkel, C.K., The projections of principal cells of the medial nucleus of the trapezoid body in the cat, J. Comp. Neurol., 238 (1985) 249-262.

87 Spangler, K.M., Cant, N.B., Henkel, C.K., Farley, G.R. and Warr, W.B., Descending projections from the superior olivary complex to the cochlear nucleus of the cat, J. Comp. Neurol., 259 (1987) 452-465. 
88 Stotler, W.A., An experimental study of the cells and connections of the superior olivary complex of the cat, $J$. Comp. Neurol., 98 (1953) 401-432.

89 Tachibana, M. and Kuriyama, K., $\gamma$-Aminobutyric acid in the lower auditory pathway of the guinea pig, Brain Research, 69 (1974) 370-374.

90 Tapia, R., $\gamma$-Aminobutyric acid: metabolism and biochemistry of synaptic transmission. In A. Lajtha (Ed.), Handbook of Neurochemistry, Vol. 3, Plenum, New York, 1983 , p. 421.

91 Thompson, G.C., Cortez, A.M. and Lam, D.M.-K., Localization of GABA immunoreactivity in the auditory brainstem of guinea pigs, Brain Research, 339 (1985) 119-122.

92 Thompson, G.C., Cortez, A.M. and Igarashi, M., GABA-like immunoreactivity in the squirrel monkey organ of Corti, Brain Research, 372 (1986) 72-79.

93 Tsuchitani, C. and Boudreau, J.C., Single unit analysis of cat superior olive S-segment with tonal stimuli, J. Neurophysiol., 29 (1966) 684-697.

94 Tolbert, L.P.,, Morest, D.K. and Yurgelun-Todd, D.K., The neuronal architecture of the anteroventral cochlear nucleus of the cat in the region of the cochlear nerve root: horseradish peroxidase labelling of identified cell types, Neuroscience, 7 (1982) 3031-3052.

95 Van Gehuchten, A., Le System Nerveux de l'Homme, Ed. 4, Trois Rois Louvain, 1906.

96 Warr, W.B., Fiber degeneration following lesions in the anterior ventral cochlear nucleus of the cat, Exp. Neurol., 14 (1966) 453-474.

97 Warr, W.B., Fiber degeneration following lesions in the posteroventral cochlear nucleus, Exp. Neurol., 23 (1969) 140-155.

98 Warr, W.B., Fiber degeneration following lesions in the multipolar and globular cell areas in the ventral cochlear nucleus of the cat, Brain Research, 40 (1972) 247-270.

99 Warr, W.B., Parallel ascending pathways from the cochlear nucleus: neuroanatomical evidence of functional specialization, Contrib. Sensory Physiol., 7 (1982) 1-38.
100 Warr, W.B., Guinan, J.J. and White, J.S., Organization of the efferent fibers: the lateral and medial olivocochlear systems. In R.A. Altschuler, R.P. Bobbin and D.W. Hoffman (Eds.), Neurobiology of Hearing: the Cochlea, Raven Press, New York, 1986, pp. 333-348.

101 Wenthold, R.J., Zempel, J.M., Parakkal, M.H., Reeks, K.A. and Altschuler, R.A., Immunocytochemical localization of GABA in the cochlear nucleus of the guinea pig, Brain Research, 380 (1986) 7-18.

102 Wenthold, R.J., Huie, D., Altschuler, R.A. and Reeks, K.A., Glycine immunoreactivity localized in the cochlear nucleus and superior olivary complex, Neuroscience, 2 (1987) 897-912.

103 Werman, R, and Aprison, M.H., Glycine: the search for a spinal cord inhibitory transmitter. In C. von Euler, S. Skoglund and U. Soderberg (Eds.), Function of Inhibitory Neuronal Mechanisms, Pergamon, New York, 1966, pp. 473-486.

104 White, J.S., Fine structure of the lateral superior olivary nucleus in the albino rat, Soc. Neurosci. Abstr., 9 (1983) 765.

105 Whitley, J.M. and Henkel, C.K., Topographical organization of the inferior collicular projection and other connections of the ventral nucleus of the lateral lemniscus in the cat, J. Comp. Neurol., 229 (1984) 257-270.

106 Yin, T.C.T., Personal communication.

107 Yin, T.C.T. and Kuwada, S., Neuronal mechanisms of binaural interaction. In G.M. Edelman, W.E. Gall and W.M. Cowan (Eds.), Dynamic Aspects of Neocortical Function, Wiley, New York, 1984, pp. 263-313.

108 Yin, T.C.T., Chan, J.C.K. and Charney, L.H., Neural mechanisms of interaural time sensitivity in the cat's auditory brainstem nuclei, International Union of Physiological Science Satellite Symposium on Hearing, University of California, San Fransicso, CA, U.S.A., 1986, p. 61.

109 Zook, J.M. and DiCaprio, R., Intracellular labeling of afferents to the lateral superior olive in the bat Eptesicus fuscus, Hearing Res., 34 (1988) 141-148. 